\title{
Overview on Interference Management Technology for Ultra-Dense Network
}

\author{
Danqing Zhang, Xinji Tian \\ School of Physics \& Electronic Information Engineering, Henan Polytechnic University, Jiaozuo, China \\ Email: tian215216@sohu.com
}

How to cite this paper: Zhang, D.Q. and Tian, X.J. (2018) Overview on Interference Management Technology for Ultra-Dense Network. Open Access Library Journal, 5: e4675.

https://doi.org/10.4236/oalib.1104675

Received: May 23, 2018

Accepted: June 17, 2018

Published: June 20, 2018

Copyright $\odot 2018$ by authors and Open Access Library Inc.

This work is licensed under the Creative Commons Attribution International License (CC BY 4.0).

http://creativecommons.org/licenses/by/4.0/

(c) (i) Open Access

\begin{abstract}
The explosive growth of the mobile traffic demand has triggered the investigation of 5th generation mobile networks $(5 \mathrm{G})$ for the future development of wireless communications. The ultra-dense network (UDN) has the ability to effectively improve system throughput and spectrum utilization by deploying a large number of low-power, low-cost, low-power home base stations or micro base stations. The dense distribution of micro base stations poses new challenges in terms of energy loss and interference management. Firstly, the overview of $5 \mathrm{G}$ is briefly introduced in this paper. Then, interference management method for UDN is summarized. So far, interference management methods for UDN mainly include interference alignment, clustering, and resource allocation. The principles of the three methods are described in detail. Finally, we conclude that clustering and resource allocation are more suitable for UDN.
\end{abstract}

\section{Subject Areas}

Mobile and Portable Communications Systems

\section{Keywords}

Ultra-Dense Network, Interference Management, Interference Alignment, Clustering, Resource Allocation

\section{Introduction}

With the popularization of portable mobile smart devices, mobile data services have shown an explosive growth trend, and a large number of hot spots have been formed in cities, posing huge challenges to existing communication systems. Although system capacity and transmission rate of $4 \mathrm{G}$ have increased dramatically compared to $3 \mathrm{G}$, it still cannot meet the rapid development of mo- 
bile data services. With the increasing demand for broadband mobile data, the next-generation mobile communication system (5G) is in urgent need to meet user needs.

Compared with 4G, 5G has a great improvement in terms of transmission rate, user experience rate, terminal-to-terminal delay, number of connected devices, mobility, and standby time in different service scenarios. According to these key technical indicators, in order to respond to the needs of future 5G network service development and maintenance operations, comprehensive consideration must be taken from various aspects such as wireless spectrum, wireless access technologies, and network architecture. The requirements of spectrum resource for future network technologies can be met by the deep development and utilization of high frequency bands and even ultra-high frequency bands. The utilization of wireless spectrum resources can also be improved by a series of advanced wireless transmission technologies. Among them, millimeter wave, massive MIMO and ultra dense networks (UDN) are regarded as the most promising key technologies for improving spectrum utilization, increasing system capacity, and enhancing $5 \mathrm{G}$ network performance.

In the current frequency allocation, it is difficult to find frequencies below 3 $\mathrm{GHz}$ that can meet future business requirements. Millimeter waves are electromagnetic waves with frequencies between $30-300 \mathrm{GHz}$. There are still many idle frequencies in these high-frequency bands, which is an important resource for expanding the available spectrum. The millimeter wave has strong directionality, which can reduce the interference between beams. However, the path loss is very large due to the high frequency characteristics of the millimeter wave. In addition, the propagation distance is limited due to the influence of the transmission environment.

Massive MIMO is also one of the most prominent technologies for increasing system capacity. The base station configures a large number of antennas and simultaneously serves several users on the same time-frequency resource. In terms of antenna configuration, these antennas can be deployed centrally on one base station to form centralized massive MIMO, and can also be distributed on multiple nodes to form distributed massive MIMO.

Massive MIMO has four advantages. Firstly, compared with the existing MIMO, the spatial resolution of massive MIMO is significantly enhanced, and spatial dimension resources can be deeply exploited. It allows multiple users in the network to use the spatial freedom provided by massive MIMO on the same time-frequency resource to communicate with the base station at the same time, which greatly improves spectrum efficiency without increasing bandwidth. Secondly, interference can be reduced by concentrating the beam in a narrow range. Thirdly, transmit power can be reduced and power efficiency can be increased [1]. Finally, when the number of antennas is large enough, the simplest linear precoding and linear detectors tend to be optimal, while noise and uncorrelated interference are negligible. 
In previous mobile communication systems, macro cell was split into multiple sectors by directional antennas, which increases the number of accessible users per unit area. However, the difficulty of obtaining the optimal base station position is also increased by the reduction of the cell area. Therefore, it is difficult to increase the number of accessible users per unit area in the 5G cell by splitting manner. Reducing the cell radius can be regarded as one of the technologies in the network architecture system for 5G. It can also increase the spatial multiplexing of spectrum resources so as to meet the demand for future data service growth. The system capacity can be improved by large number of small base stations. In the 5G network architecture, in a coverage area of a macro base station, the density of various types of small base stations will be much higher than that of existing small base stations. These small base stations have low transmission power and may use various wireless transmission technologies. Each small base station only serves a small number of terminal users or even one user. The user-intensive network served by such base stations is called an ultra-dense network.

Most of the voice and data services take place in indoor and hot spots. Heterogeneous wireless networks incorporating multiple access technologies can solve the problem of rapid traffic growth in indoor coverage and hot-pots. With the rapid increase of the number and the density of users, UDN with multiple node deployments becomes the development trend of future mobile communications. UDN improves the system capacity by deploying a large number of low-power, low-cost, low-power home base stations or micro base stations [2] [3]. The base station can be directly deployed indoors, which not only can greatly reduce signal attenuation, but also can improve the link quality between the base station and the terminal [4] [5] [6] [7] [8].

Although UDN technology has great development prospects in 5G, there are still some technical challenges that need to be resolved. Small cells with different transmission frequencies are deployed in an overlapping manner with macro cells, and the density of small cells is extremely high. The planning of frequency reuse factor greater than 1 makes the interference problem in the network more obvious [9]. Co-channel interference has become a major obstacle to UDN technology development. The existence of multi-layer networks and different access points also brings more trouble to users. How to select the best access point among multiple available networks is also a problem that needs to be solved urgently for UDN.

\section{UDN}

\subsection{Small Cell}

Small cell is a kind of wireless access node with low transmit power and small coverage. It can be used as a supplement to $4 \mathrm{G}$ or $5 \mathrm{G}$ macro cells and operates in authorized and unlicensed frequency bands. The number of users it serves is also relatively small, about 2 to 4 users, and the effective coverage is about 10 to 20 
meters. In contrast, the coverage of macro base stations can reach several kilometers. Small cell supports a variety of wireless access standards including GSM, CDMA2000, TD-SCDMA, LTE, and WiMax. Small cell is an integral part of the LTE network. In $3 \mathrm{G}$, small cells are regarded as a traffic distribution technology. In $4 \mathrm{G}$, the concept of HetNet (Heterogenous Network) is introduced. The mobile network consists of multiple layers such as small cells and macro cells. Due to the explosive growth of data traffic, data offloading has become an efficient method of using wireless spectrum resources. In hot spots, small cells can share up to $70 \%$ of traffic.

The initial form of small cell is femtocell. The home base station was originally designed to provide indoor coverage of the small station. Now its concept has also been expanded: metrocell, hot spot access femtocell, enterprise femtocell and super femtocell, and so on. The small cell now incorporates microcells, picocells, home base stations, and distributed wireless technologies to provide network coverage in a ten-meter indoor environment or a two-kilometer outdoor environment [10]. The mobile operator uses the small cell to extend the coverage of the macro base station and to increase the capacity of the communication network. In areas where there are many users and traffic is heavy, small cells can divert traffic into 80 percent.

The application of the small cell is very rich. It can be placed in the blind area of the macro base station to enhance the network coverage, or it can be placed in the hot spot of the macro base station to reduce the physical distance between the network and the terminal equipment, so as to improve the link quality and increase system capacity. The deployment of the scenario must take into account the requirement for intra-frequency or heterogeneous deployment, indoor or outdoor deployment with macro base stations.

\subsection{UDN Overview}

Since the $5 \mathrm{G}$ includes new wireless transmission technologies and the subsequent evolution of various existing wireless access technologies. A variety of wireless access technologies are adopted by 5G, such as LTE, UMTS and Wi-Fi. There are both macro stations responsible for basic coverage and low-power small stations that carry hot spot coverage, such as multi-layer coverage multi-radio access technologies such as Micro, Pico, Relay, and Femto, and heterogeneous overlay heterogeneous networks [11]. Among these large number of low-power nodes, some are planned macro nodes deployed by operators. The others are likely to be deployed by users without planned low-power nodes. These low-power nodes may be of the Open Subscriber Group (OSG) type or may be of the Closed Subscriber Group (CSG) type, which make the network topology and features extremely complex.

In ultra-dense network, the throughput can be increased by reducing the path loss between the base station and the user terminal. This makes the system amplify the interference signal while increasing the effective reception signal. Fur- 
ther, the dramatic increase in cell density makes the interference extremely complex [12]. Therefore, how to effectively eliminate interference is an issue that needs to be considered for ultra-dense network.

\section{Interference Management}

The interference management in UDN includes interference alignment, clustering, resource allocation and so on.

\subsection{Interference Alignment}

Interference Alignment (IA) is an effective interference management mechanism. Interference can be overlapped at the receiver through IA pre-coding. Thus, interference can be completely separated from the desired signals. IA scheme include two types: centralized IA and distributed IA.

In centralized IA, interference-aligned pre-coding matrices or decoding matrices are calculated according to at global channel state information obtained at central controller. Central controller sends this matrices to each Access Point (AP) and user. The central controller can be any AP or special functional entity outside the AP. The centralized IA is suitable for backhaul deployment scenario and TDD duplex mode between APs. Utilizing channel heterogeneity, AP first performs uplink channel estimation, and then sends some of its estimated partial channel information to the central controller.

In distributed IA, pre-coding/decoding matrixes are calculated independently at each AP and then are send to subordinate UE. This scheme is suitable for the scenario of no backhaul deployment between APs. Each AP needs to obtain global channel state information, and the overhead of acquiring channel state information in TDD and FDD modes is relatively large. This means that IA can achieve better performance in channel-grading scenarios, such as indoor or outdoor transmission between low mobility or stationary user equipment.

\subsection{Clustering}

There are a large number of various wireless access nodes in the high density scenario. It is difficult for the centralized control mode to handle a large amount of computation [13]. The clustering of the network topology and the resource allocation in units of clusters have become an important means of improving network performance [14].

The whole network is divided into several small networks through clustering. Each small network includes multiple micro base stations that are multiple small cells. Cluster head is selected from multiple micro base stations in each cluster, which is responsible for the resource allocation within this cluster. Clustering can reduce the network size, reduce the amount of computation, and improve the efficiency and performance of network operations [15] [16] [17] [18].

At present, the research on clustering algorithms mostly focuses on graph theory and matrix. The nodes, the edge between nodes and the weight of edge 
denote the micro base station, the interference between small cells and the interference value. The graph is decomposed into multiple sub-graphs using MAX-K-CUT, K-means and other algorithms. Each node in the sub-graph is a cluster. Select a node as the cluster head, which act as the control node responsible for scheduling the cluster resource and sending other control signaling. Each cluster head only needs to deal with the scheduling problem within the cluster, which reduces the network size and the amount of operations to be processed. The matrix-based clustering algorithm counts the interference between two micro base stations or any two users. These interference values are combined to form a matrix. Clusters are selected from the matrix in turn with the goal of maximizing interference [19] [20].

The idea of clustering is classified into two categories. One is to divide the micro base stations with very little interference between each other into one cluster, and to place the micro base stations with larger interference into different clusters. After clustering, each cluster is assigning a sub-channel. In [21], Sun Y, et al. have proposed a multi-cluster based dynamic channel assignment (MC-DCA) and in [22], Kim S J have proposed a Clustering-based Co-tier Interference Coordination (CCIC) algorithm. Both of these two algorithms adopt the graph dyeing theory, which classifies the base stations with small interference into the same cluster and the base stations with large interference into different clusters. However, this clustering scheme needs to allocate different resources to different clusters. With the sudden increase of base stations, resources are inadequate. In [23], Chen L has proposed a clustering strategy based on similarity, which defines the reciprocal of the path loss between two base stations as the similarity between two cells. The cluster heads were selected by the max-sum algorithm [24] and then micro base stations are clustered to maximize the cluster similarity.

The other clustering idea is to divide the base stations with very large interference between each other into a cluster. So the interference of different clusters is very small. Cluster head first guarantees the communication quality of the members in the cluster, and then suppress the inter-cluster interference. Most of the studies on interference management are based on this idea. In [25], Kschischang F R, et al. use the idea of game theory to group base stations with large interference into the same cluster. In [26], Liu L, et al. use a modified K-means algorithm to cluster, and in [27], Wei R, et al. use subtraction clustering to find the cluster heads.

Most clustering algorithms use small cells as the unit to perform clustering on the micro base station. Some clustering algorithms also cluster users of small cells or perform clustering with load information [28] [29] [30] [31] [32]. For example, in [33], an edge is set between every two users, the weight of the edge is determined by the interference between users, and then clustering is performed using graph theory so that the sum of the interference in each cluster is minimized and ensures that users within the cluster do not have significant interference. In [34], Pateromichelakis E have proposed a user-centric clustering algo- 
rithm based on load information, taking the load information as a clustering consideration.

Using clustering algorithm to segment the network and using cluster heads for resource allocation can effectively eliminate intra-cluster interference, but inter-cluster interference is still a problem in clustering technology. Inter-cluster interference occurs because some cells may interfere with members of multiple clusters. However, in a traditional clustering system, each cell is allowed to join only one cluster, which inevitably interferes with other clusters. The literature [35] allows a community to participate in multiple clusters, coordinate inter-cluster and intra-cluster interference, and establish a coalition formation game model using cooperative game theory. The players in each cluster contributes their own resources to form resource pools, and then obtains resources from the cluster according to the degree of contribution to communicate. The cells participating in multiple clusters divide the available frequencies into several parts, contribute to different clusters and acquire certain resources from each cluster at the same time. This clustering idea that allows each cluster to cross can be more effectively allocate resources among clusters and avoid inter-cluster interference.

\subsection{Resource Allocation}

Resource allocation is an important issue in wireless communication. The core resources in a wireless communication system are frequency and power. By reasonably allocating frequency resources and performing power control, it can effectively use limited communication resources and provide users with better service.

Resource management includes frequency allocation, resource block allocation, power allocation and time allocation [36]. There are three ways to allocate frequency between small cells and macro cells: orthogonal allocation, full multiplexing, and partial multiplexing [37]. Under the orthogonal allocation mode, macro cells and small cells use different frequency, which can effectively eliminate inter-layer interference. However, this way of dedicated frequency will reduce spectrum efficiency and affect system capacity. In full multiplexing mode, the small cell can use all the frequency resources of the macro cell. So the frequency reuse rate is high. However there may be serious interference. In partial multiplexing mode, the frequency resources of the macro cell are divided into two parts. One is a dedicated for macro cell, and the other is a dedicated for both macro cell and small cell. In terms of frequency allocation, orthogonal frequency division multiplexing is a key technology used in next generation wireless communications. It has the ability to guarantee the orthogonality among users. So interference can be effectively avoided. It is also possible to flexibly select suitable sub-carrier for transmission to achieve dynamic frequency domain resource allocation.

Frequency allocation between small cells includes full multiplexing and partial 
multiplexing [38]. In full multiplexing mode, all small cells use the same frequency band and there is serious interference. Partial multiplexing often uses dyeing theory to allocate frequency. In dyeing theory, interference graphs are established by treating small cells or users as nodes. Then, color each node so as to the colors of adjacent nodes are different. Assign the same frequency band to nodes of the same color, and assign different frequency bands to nodes of different colors [39].

Resource block allocation, that is, sub-channel allocation, can eliminate inter-layer interference and intra-layer interference. Allocating orthogonal resource blocks for macro base stations and micro base stations can eliminate inter-layer interference. Allocating resource blocks within a cluster or between clusters can eliminate some intra-layer interference. If inter-cluster interference is larger than intra-cluster interference, allocate orthogonal resource block to different clusters, or allocate resource blocks based on graph dyeing theory. The former is orthogonal allocation, and the latter is partial reuse [40] [41] [42] [43] [44]. If intra-cluster interference is larger than inter-cluster interference, orthogonal resource blocks are allocated to users in each cluster.

When sub-channels are allocated to the micro base station, it is necessary to ensure that the SUE cannot affect the normal communication of macro user. The interference caused to the MUE must be restricted to a certain SINR threshold. At the same time, the interference between the small cells in the same layer must also meet a certain threshold. In an actual communication system, due to multi-path and shadow fading of a wireless link, there are many factors that can be considered for sub-channel allocation, such as the highest-gain sub-channel, the SINR of each user, and so on.

The power allocation includes the user's power allocation and the power allocation of resource blocks. There are two modeling methods for power distribution. One is the water filling algorithm, which allocates more power to users or resource blocks with high channel gains, and allocates less power to users or resource blocks with low channel gains, even without allocating power [45]. The water injection algorithm can make full use of the available power to increase the system capacity. However, fairness is not taken into consideration in this algorithm. Users with poor channel quality may not get normal services in extreme cases. The other is to solve the power allocation problem based on the idea of optimization, in which the cost is power and optimization objective is the difference between throughput and power [46]-[52].

Time allocation includes time allocation between macro cells and small cells and time allocation between small cells. The goal of time allocation between macro cells is to maximize the throughput. When the small cell transmits signal, the macro cell uses ABS (Almost Blank Sub-frame) to reduce the transmission power or doesn't transmit signals, which can reduce or eliminate inter-layer interference [53] [54] [55] [56]. There are two type of time allocation between small cells. One is performed by graph dyeing algorithm. The other is to divide ABS into several part allocated to each cluster or small cell. 
A mixed-integer nonlinear programming model based on the optimization method is proposed in [57], in which optimization of sub-channel allocation and power control are joint performed. The goal is to maximize the sum of the throughput of each cluster. The constraints include the total user capacity in each cluster, the total transmit power of the femtocell on each channel, inter-layer interference, and intra-layer interference level.

Using the optimization strategy to solve the resource scheduling scheme and maximizing the system performance is the most direct way to optimize the system performance. Such optimization problems can be solved with traditional convex optimization tools such as interior point method, branch delimitation method, Lagrangian multiplier method, and so on. However, due to the non-linearity of the problem and the discontinuity of the solution variables, this type of optimization problem is NP-hard [58], which causes great difficulties for the computational solution. So low-complexity resource scheduling strategies have been proposed.

Ref. [59] has proposed adaptive frequency allocation scheme, in which the available frequencies of the small cell are divided into a dedicated portion and a shared portion. According to the number of small cells, the dedicated frequency is divided into several frequency bands to ensure the basic communication connection of the small cell. Each micro base station reports and records the cell ID that interferes with the cell by the user, and records the user's gain on each sub-channel according to the history information. The number of cells that have interference with the cell is defined as the interference degree. Each cell is sorted according to the interference degree. Small cell with the highest interference degree has the highest priority, and it selects the highest gain frequency from the resources. Shared resources, used to improve QoS, are obtained by the competition of each cell according to the service needs of the cell [60]. This low-complexity resource allocation strategy requires very little computation [61]. But there is a certain gap between system performance and optimization.

In the high-density trend, distributed algorithms can effectively balance performance and complexity [62]. In [63], the authors design a micro base station using game theory and enhanced learning algorithm, and distributed algorithm based on historical revenue and frequency selection. They designed two games. The first one was a non-cooperative game, in which each small cell used its own throughput as revenue. The second one was a cooperative game, in which the revenue of all small cells was the throughput of all the small cells in the system. All micro base stations act as players, and they choose their own strategies based on historical returns to maximize revenue. This algorithm does not require the central control node to be responsible for the resource scheduling in the network. Each micro base station independent allocates frequency and power based on historical revenues to optimize its own revenue.

\section{Conclusion}

With the rapid development of communication systems and smart terminals, 
user demands for communication network capacity are exploding. UDN has become an indispensable key technology for future 5G communications. We detailedly describe three interference management methods, including IA. From the Third Section, we can conclude that clustering and resource allocation are more suitable for UDN.

\section{References}

[1] Ngo, H.Q., Larsson, E.G. and Marzetta, T.L. (2011) Energy and Spectral Efficiency of Very Large Multiuser MIMO Systems. IEEE Transactions on Communications, 61, 1436-1449.

[2] Bhushan, N., Li, J., Malladi, D., et al. (2014) Network Densification: The Dominant Theme for Wireless Evolution into 5G. Communications Magazine IEEE, 52, 82-89. https://doi.org/10.1109/MCOM.2014.6736747

[3] Marzetta, T.L. (2010) Noncooperative Cellular Wireless with Unlimited Numbers of Base Station Antennas. IEEE Transactions on Wireless Communications, 9, 3590-3600. https://doi.org/10.1109/TWC.2010.092810.091092

[4] Yang, J., Chen, L., Liu, Q., et al. (2015) Review on Key Technologies in 5G Mobile Communication System. Mobile Communications, 39, 79-84.

[5] Ye, J., Ge, X., Mao, G., et al. (2017) 5G Ultra-Dense Networks with Non-Uniform Distributed Users.

[6] Ding, M. and López-Pérez, D. (2017) On the Performance of Practical Ultra-Dense Networks: The Major and Minor Factors. IEEE International Symposium on Modeling and Optimization in Mobile, Ad Hoc, and Wireless Networks, Paris, 15-19 May 2017, 1-8.

[7] Li, P., Jiang, H., Gao, S., et al. (2016) Impact of BS Sleeping and User Association Scheme on Delay in Ultra-Dense Networks. IEEE International Conference on Wireless Communications \& Signal Processing, Yangzhou, 13-15 October 2016, 1-6.

[8] Feng, M. and Mao, S. (2017) Interference Management and User Association for Nested Array-Based Massive MIMO HetNets. IEEE Transactions on Vehicular Technology, 99, 1-1.

[9] Galiotto, C., Marchetti, N. and Doyle, L. (2012) Flexible Spectrum Sharing and Interference Coordination for Low Power Nodes in Heterogeneous Networks. IEEE Vehicular Technology Conference, Quebec City, QC, 3-6 September 2012, 1-5.

[10] Lin, Z.S. (2014) Stochastic Geometry Analysis on Small Cell Networks and Enhanced Technologies. Beijing University of Posts and Telecommunications, Beijing.

[11] Osseiran, A., Braun, V., Hidekazu, T., et al. (2014) The Foundation of the Mobile and Wireless Communications System for 2020 and Beyond: Challenges, Enablers and Technology Solutions. IEEE Vehicular Technology Conference, 1-5.

[12] Dat, P.T., Kanno, A., Yamamoto, N., et al. (2016) 5G Transport Networks: The Need for New Technologies and Standards. IEEE Communications Magazine, 54, 18-26. https://doi.org/10.1109/MCOM.2016.7565268

[13] Chen, S.Y., Xing, C.W. and Fei, Z.S. (2015) Distributed Resource Allocation in U1tra-Dense Networks via Belief Propagation. China Communication, 12, 79-91.

[14] Cho, M.J., Ban, T.W., Bang, C.J., et al. (2015) A Distributed Scheduling with Interference-Aware Power Control for Ultra-Dense Networks. IEEE International Conference on Communications, London, 8-12 June 2015, 1661-1666.

[15] Chen, S., Xing, C., Fei, Z., et al. (2016) Dynamic Clustering Algorithm Design for 
Ultra Dense Small Cell Networks in 5G. IEEE International Conference on Communications and Networking in China, 836-840.

[16] Tang, S., Sun, C., Wang, J., et al. (2016) Interference Management Based on Cell Clustering in Ultra-Highly Dense Small Cell Networks. International Conference on Information and Communications Technologies, Xi'an, 24-26 April 2015, 1-6.

[17] Zhao, C., Xu, X., Gao, Z. and Huang, L. (2016) A Coloring-Based Cluster Resource Allocation for Ultra Dense Network. IEEE International Conference on Signal Processing, Communications and Computing, Hong Kong, 5-8 August 2016, 1-5.

[18] Zhang, H., Li, H., Lee, J.H. and Dai, H.Y. (2017) QoS-Based Interference Alignment with Similarity Clustering for Efficient Subchannel Allocation in Dense Small Cell Networks. IEEE Transactions on Communications, 65, 5054-5066. https://doi.org/10.1109/TCOMM.2017.2737001

[19] Qin, C. and Tian, H. (2014) A Greedy Dynamic Clustering Algorithm of Joint Transmission in Dense Small Cell Deployment. Consumer Communications and Networking Conference, Las Vegas, 10-13 January 2014, 629-634.

[20] Seno, R., Ohtsuki, T., Jiang, W., et al. (2016) A Low-Complexity Cell Clustering Algorithm in Dense Small Cell Networks. EURASIP Journal on Wireless Communications and Networking, 2016, 262. https://doi.org/10.1186/s13638-016-0765-3

[21] Sun, Y., Chang, Y., Hu, M. and Wang, B. (2015) A Cluster-Based Hybrid Access Strategy Using Non-Cooperative Game Theory for Ultra-Dense HetNet. IEEE International Conference on High PERFORMANCE Computing and Communications, New York, 24-26 August 2015, 14-19. https://doi.org/10.1109/HPCC-CSS-ICESS.2015.146

[22] Kim, S.J., Cho, I.K., Lee, B.B., Bae, S.-H. and Cho, C.-H. (2016) Multi-Cluster based Dynamic Channel Assignment for Dense Femtocell Networks. KSII Transactions on Internet \& Information Systems, 10, 1535-1554.

[23] Chen, L., Xia, H., Feng, C. and Wu, S. (2015) Clustering-Based Co-Tier Interference Coordination in Dense Small Cell Networks. IEEE 26th Annual International Symposium on Personal, Indoor, and Mobile Radio Communications (PIMRC), Hong Kong, 30 August-2 September 2015, 1878-1882. https://doi.org/10.1109/PIMRC.2015.7343605

[24] Hosseini, K., Dahrouj, H. and Adve, R. (2012) Distributed Clustering and Interference Management in Two-Tier Networks. Global Communications Conference, Anaheim, 3-7 December 2012, 4267-4272.

[25] Kschischang, F.R., Frey, B.J. and Loeliger, H.-A. (2001) Factor Graphs and the Sum-Product Algorithm. IEEE Transactions on Information Theory, 47, 498-519. https://doi.org/10.1109/18.910572

[26] Liu, L., Garcia, V., Tian, L., et al. (2015) Joint Clustering and Inter-Cell Resource Allocation for CoMP in Ultra Dense Cellular Networks. IEEE International Conference on Communications, London, 8-12 June 2015, 2560-2564. https://doi.org/10.1109/ICC.2015.7248710

[27] Wei, R., Wang, Y. and Zhang, Y. (2015) A Two-Stage Cluster-Based Resource Management Scheme in Ultra-Dense Networks. IEEE/CIC International Conference on Communications in China, Shanghai, 13-15 October 2014, 738-742.

[28] Qiu, J., Ding, G., Wu, Q., et al. (2017) Hierarchical Resource Allocation Framework for Hyper-Dense Small Cell Networks. IEEE Access, 4, 8657-8669. https://doi.org/10.1109/ACCESS.2016.2633434

[29] Liu, Y., Li, X., Ji, H., et al. (2017) Grouping and Cooperating among Access Points in User-Centric Ultra-Dense Networks with Non-Orthogonal Multiple Access. 
IEEE Journal on Selected Areas in Communications, 35, 2295-2311. https://doi.org/10.1109/JSAC.2017.2724680

[30] Ye, Y., Zhang, H., Xiong, X. and Liu, Y. (2015) Dynamic Min-Cut Clustering for Energy Savings in Ultra-Dense Networks. Vehicular Technology Conference, Boston, MA, 6-9 September 2015, 1-5. https://doi.org/10.1109/VTCFall.2015.7390904

[31] Lin, Y., Zhang, R., Yang, L., et al. (2017) Secure User-Centric Clustering for Energy Efficient Ultra Dense Networks: Design and Optimization. IEEE Journal on Selected Areas in Communications, PP, 1-1.

[32] Sun, G., Zhang, H. and Liu, G. (2016) User Demand Aware Soft-Association Control in Ultra-Dense Small Cell Networks. IEEE 41 st Conference on Local Computer Networks, Dubai, 7-10 November 2016, 651-654.

[33] Sun, W. and Teng, Y. (2017) Impact of User Mobility on Transmit Power Control in Ultra Dense Networks. IEEE International Conference on Communications Workshops, Paris, 21-25 May 2017, 1165-1170.

[34] Pateromichelakis, E., Shariat, M., Quddus, A., et al. (2013) Dynamic Clustering Framework for Multi-Cell Scheduling in Dense Small Cell Networks. IEEE Communications Letters, 17, 1802-1805.

https://doi.org/10.1109/LCOMM.2013.072313.131248

[35] Cao, J., Peng, T., Qi, Z., et al. (2018) Interference Management in Ultra-Dense Networks: A User-Centric Coalition Formation Game Approach. IEEE Transactions on Vehicular Technology, PP, 1-1.

[36] Chuang, M.C., Chen, M.C. and Lin, Y.H. (2017) SDN-Based Resource Allocation Scheme in Ultra-Dense OFDMA Smallcell Networks. International Conference on Advanced Materials for Science and Engineering, Tainan, 12-13 November 2016, 524-527.

[37] Gathala, S.A.K., Dhurjati, D., Ermolinskiy, A. and Vick, C.A. (2015) Energy-Efficient Run-Time Offloading of Dynamically Generated Code in Heterogenuous Multiprocessor Systems. US Patent No. 13961122.

[38] Lv, H., Xia, X., Hua, Z. and Yu, Y. (2015) Researching the Key Technologies of Wireless Sensor Network Node in Power Distribution Room Status Monitoring. International Conference on Cloud Computing and Internet of Things, Changchun, 13-14 December 2014, 161-165.

[39] Huang, J., Zhou, P., Teng, D., et al. (2017) Clustering-Based Energy-Saving Algorithm in Ultra-Dense Network. IOP Conference Series: Earth and Environmental Science, 012145.

[40] Du, J., Gelenbe, E., Jiang, C., et al. (2017) Contract Design for Traffic Offloading and Resource Allocation in Heterogeneous Ultra-Dense Networks. IEEE Journal on Selected Areas in Communications, 35, 2457-2467. https://doi.org/10.1109/JSAC.2017.2760459

[41] Chen, M., Hua, Y., Gu, X., et al. (2016) A Self-Organizing Resource Allocation Strategy Based on Q-Learning Approach in Ultra-Dense Networks. IEEE International Conference on Network Infrastructure and Digital Content, Beijing, 23-25 September 2016, 155-160. https://doi.org/10.1109/ICNIDC.2016.7974555

[42] Liu, Y., Wang, Y., Zhang, Y., et al. (2016) Game-Theoretic Hierarchical Resource Allocation in Ultra-Dense Networks. IEEE International Symposium on Personal, Indoor, and Mobile Radio Communications, Valencia, 4-8 September 2016, 1-6. https://doi.org/10.1109/PIMRC.2016.7794819

[43] Hua, C., Luo, Y. and Liu, H. (2016) Wireless Backhaul Resource Allocation and Us- 
er-Centric Clustering in Ultra-Dense Wireless Networks. IET Communications, 10, 1858-1864. https://doi.org/10.1049/iet-com.2016.0119

[44] Cheng, L., Gao, Y., Li, Y., et al. (2015) A Cooperative Resource Allocation Scheme Based on Self-Organized Network in Ultra-Dense Small Cell Deployment. IEEE 81 st Vehicular Technology Conference, Glasgow, 11-14 May 2015, 1-6. https://doi.org/10.1109/VTCSpring.2015.7145594

[45] Shen, S. and Lok, T.M. (2013) Dynamic Power Allocation for Downlink Interference Management in a Two-Tier OFDMA Network. IEEE Transactions on Vehicular Technology, 62, 4120-4125. https://doi.org/10.1109/TVT.2013.2262955

[46] Gao, Y., Cheng, L., Zhang, X., et al. (2016) Enhanced Power Allocation Scheme in Ultra-Dense Small Cell Network. China Communication, 13, 21-29.

[47] Xu, L., Mao, Y., Leng, S., et al. (2017) Energy-Efficient Resource Allocation Strategy in Ultra Dense Small-Cell Networks: A Stackelberg Game Approach. IEEE International Conference on Communications, Paris, 21-25 May 2017, 1-6. https://doi.org/10.1109/ICC.2017.7997289

[48] Zhang, T., Zhao, J., An, L., et al. (2016) Energy Efficiency of Base Station Deployment in Ultra Dense HetNets: A Stochastic Geometry Analysis. IEEE Wireless Communications Letters, 5, 184-187. https://doi.org/10.1109/LWC.2016.2516010

[49] De Mari, M. and Quek, T. (2017) Energy-Efficient Proactive Scheduling in Ultra Dense Networks. IEEE International Conference on Communications, Paris, 21-25 May 2017, 1-6. https://doi.org/10.1109/ICC.2017.7996471

[50] Mwanje, S.S. and Ali-Tolppa, J. (2016) Fluid Capacity for Energy Saving Management in Multi-Layer Ultra-Dense 4G/5G Cellular Networks. International Conference on Network and Service Management, Montreal, 31 October-4 November 2016, 233-237.

[51] Zhang, H. (2016) Research of Area Spectrial Efficiency and Area Energy Efficiency in Ultra-Dense Networks. Harbin Institute of Technology, Harbin.

[52] Kao, H., Wei, C.Y., Lin, H.C., et al. (2016) Adaptive Measurement for Energy Efficient Mobility Management in Ultra-Dense Small Cell Networks. IEEE International Conference on Communications, Kuala Lumpur, 22-27 May 2016, 1-6.

[53] Singh, R. and Murthy, C.S.R. (2016) Techniques for Interference Mitigation Using Cooperative Resource Partitioning in Multitier LTE HetNets. IEEE Systems Journal, 12, 843-853. https://doi.org/10.1109/JSYST.2016.2527504

[54] Huang, P.H., Kao, H. and Liao, W. (2017) Cross-Tier Cooperation for Optimal Resource Utilization in Ultra-Dense Heterogeneous Networks. IEEE Transactions on Vehicular Technology, 66, 11193-11207. https://doi.org/10.1109/TVT.2017.2732165

[55] Xu, J., Cheng, J., Han, L. and Zhou, J. (2013) Lifetime Scaling Law of Ordinary Clustering Ultra-Wideband Sensor Network. Wireless Communications and Mobile Computing Conference, Sardinia, 1-5 July 2013, 1464-1469. https://doi.org/10.1109/IWCMC.2013.6583772

[56] Zhang, Z., Ma, Z., Xiao, M., et al. (2016) Two-Timeslot Two-Way Full-Duplex Relaying for $5 \mathrm{G}$ Wireless Communication Networks. IEEE Transactions on Communications, 64, 2873-2887. https://doi.org/10.1109/TCOMM.2016.2574845

[57] Abdelnasser, A. and Hossain, E. (2013) Subchannel and Power Allocation Schemes for Clustered Femtocells in Two-Tier OFDMA HetNets. IEEE International Conference on Communications Workshops, Budapest, 9-13 June 2013, 1129-1133. https://doi.org/10.1109/ICCW.2013.6649406

[58] Yan, R., Zhang, G., Li, B., et al. (2013) An Effective Semi-Static Interference Coor- 
dination Scheme for Wireless Cellular Systems. Wireless Telecommunications Symposium, Phoenix, AZ, 17-19 April 2013, 1-6.

[59] Wu, Y., Jiang, H. and Zhang, D. (2012) A Novel Coordinated Spectrum Assignment Scheme for Densely Deployed Enterprise LTE Femtocells. IEEE 75 th Vehicular Technology Conference, Yokohama, 6-9 May 2012, 1-6. https://doi.org/10.1109/VETECS.2012.6239854

[60] Mahmood, N.H., Berardinelli, G., Pedersen, K.I. and Mogensen, P. (2015) An Interference-Aware Distributed Transmission Technique for Dense Small Cell Networks. IEEE International Conference on Communication Workshop, London, 8-12 June 2015, 119-124.

[61] Soldati, P. and Koudouridis, G.P. (2015) Distributed Spectrum Access in Dense 5G Networks. IEEE International Symposium on Personal, Indoor, and Mobile Radio Communications, Hong Kong, 30 August-2 September 2015, 1901-1906.

https://doi.org/10.1109/PIMRC.2015.7343609

[62] Oueis, J., Strinati, E.C., Sardellitti, S., et al. (2016) Small Cell Clustering for Efficient Distributed Fog Computing: A Multi-User Case. IEEE 82nd Vehicular Technology Conference, Boston, MA, 6-9 September 2015, 1-5.

[63] Bennis, M., Perlaza, S.M., Blasco, P., et al. (2013) Self-Organization in Small Cell Networks: A Reinforcement Learning Approach. IEEE Transactions on Wireless Communications, 12, 3202-3212. https://doi.org/10.1109/TWC.2013.060513.120959 